\title{
Bose-Einstein Condensation of Confined Atomic Gases at Ultra Low Temperatures
}

\author{
M. Serhan ${ }^{1}$ \\ ${ }^{1}$ Department of Physics, Faculty of Science, Al al-Bayt University, Al-Mafraq, Jordan \\ Correspondence: M. Serhan, Department of Physics, Faculty of Science, Al al-Bayt University, Al-Mafraq, Jordan. \\ E-mail: mserhan@aabu.edu.jo
}

Received: August 26, 2017

Accepted: September 18, 2017 Online Published: September 23, 2017

doi:10.5539/apr.v9n5p96

URL: https://doi.org/10.5539/apr.v9n5p96

\begin{abstract}
In this work I solve the Gross-Pitaevskii equation describing an atomic gas confined in an isotropic harmonic trap by introducing a variational wavefunction of Gaussian type. The chemical potential of the system is calculated and the solutions are discussed in the weakly and strongly interacting regimes. For the attractive system with negative scattering length the maximum number of atoms that can be put in the condensate without collapse begins is calculated
\end{abstract}

\section{Introduction}

Bose-Einstein condensation (Pethick \& Smith, 2008) is the macroscopic occupation of the lowest momentum state with a finite fraction of particles. This phenomenon had interested both experimental and theoretical physicists from the beginning of the last century since Bose and Einstein conjectured the existing of this phenomenon in the free Bose gas. The first experimental achievement of it came before twenty two years by different experimental groups (Anderson et al., 1995; Davis et al., 1995; Bradley et al., 1995). This achievement had increased the interest in analyzing different aspects related to this phenomenon (Baym \& Pethick, 1996; Fetter, 2009; Mateo \& Delgado, 2007).

Different theoretical approaches have been used to study these systems that undergoes Bose-Einstein condensation. These methods include mean-field theories (Margetis, 2015), canonical and microcanonical ensemble approaches (Chase, Mekjian, \& Zamick, 1999), and numerical calculations including quantum Monte-Carlo techniques (DuBois \& Glyde, 2001).

These systems are weakly-interacting ultracold atomic gases confined in an external trap. A good description of these systems is accomplished by the Gross-Pitaevskii equation (Rogel-Salazar, 2013) which is effectively a nonlinear Schrodinger equation. In three dimensions this equation takes the form:

$$
-\frac{\hbar^{2}}{2 m} \nabla^{2} \psi+U \psi+N_{0} g|\psi|^{2} \psi=\mu \psi
$$

where the first term is the kinetic energy, $\mathrm{U}$ is the external trap potential and the third term is the nonlinear term arises from the interaction between atoms and $\mu$ is the chemical potential and $g=\left(4 \pi \hbar^{2} \mathrm{a} / \mathrm{m}\right)$, a being the scattering length. We take the external trap potential to be isotropic and simple harmonic:

$$
U(r)=(1 / 2) m \omega r^{2}
$$

where $m$ is the mass of the atom and $\omega$ the angular frequency

The approach in this work will be variational. In the next section we make an appropriate ansatz of a trial variational wavefunction of Gaussian type which will be followed by the calculation of the ground state energy which consists of three parts.

\section{Variational Wavefunction}

In the absence of interactions between the atoms the ground state wave function is Gaussian. Interactions will certainly modify both the wave function and energy. As a trial wavefunction we use a Gaussian function of the form 


$$
\psi=A e^{-b r^{2}}
$$

Where $b$ is our variational parameter. Normalization of the wavefunction gives $A=(2 b / \pi)^{3 / 4}$

Using the normalized form of the wavefunction to calculate the ground state energy we get first for the kinetic energy:

$$
\langle T\rangle=-\frac{\hbar^{2}}{2 m} \int \psi \nabla^{2} \psi d^{3} r=\frac{3 \hbar^{2}}{2 m}
$$

For the expectation value of the harmonic potential we get:

$$
\langle U\rangle=(1 / 2) m \omega^{2} \int r^{2} \psi^{2} d^{3} r=\frac{3 m \omega^{2}}{8 b},
$$

and finally for the expectation value of the interaction term we get

$$
\langle V\rangle=g \int \psi^{4} d^{3} r=g\left(\frac{b}{\pi}\right)^{3 / 2} .
$$

Therefore we get for the chemical potential

$$
\mu=\frac{3 \hbar^{2} b}{2 m}+\frac{3 m \omega^{2}}{8 b}+N_{0} g\left(\frac{b}{\pi}\right)^{3 / 2}
$$

Now if we define the dimensionless quantities $B=\frac{\hbar b}{m \omega}$ and $\mu^{*}=\frac{\mu}{\hbar \omega}$ eq.(7) is reduced to

$$
\mu^{*}=\frac{3}{2} B+\frac{3}{8 B}+\chi B^{3 / 2}
$$

where $\chi=N_{0}(m \omega / \pi \hbar)^{1 / 2} a$.

Minimization of $\mu^{*}$ gives:

$$
\frac{d \mu^{*}}{d B}=0=\frac{3}{2}-\frac{3}{8 B^{2}}+\frac{3}{2} \chi B^{1 / 2}
$$

or equivalently

$$
B^{2}-1 / 4+\chi B^{5 / 2}=0
$$

\section{Results and Discussion}

In eq.(10) if $\chi=0$, we obtain $B=1 / 2$ or $(b=\mathrm{m} \omega / 2 \hbar)$ which is the ground state of the harmonic trap with chemical potential $3 / 2 \hbar \omega$. For $\chi<<1$, we get $B=\frac{1}{2}\left(1-\frac{\chi}{2 \sqrt{2}}\right)$, in this case we obtain for the chemical potential $\mu=\hbar \omega\left(\frac{3}{2}+\frac{\chi}{2 \sqrt{2}}\right)$

Thus a small repulsive scattering length increases the energy, whereas a small attractive scattering length lowers the energy.

For $\chi>>1$, we get $B \approx(1 / 4 \chi)^{2 / 5}\left(1-\frac{8}{5}\left[\frac{1}{4 \chi}\right]^{4 / 5}\right)$. Thus the chemical potential equals in this limit

$$
\mu \cong \frac{5}{8}(4 \chi)^{2 / 5} \hbar \omega\left(1-\frac{96}{25}(4 \chi)^{-8 / 5}\right)
$$

In this case the kinetic energy is much smaller than the trapping potential energy.

$$
\frac{\langle T\rangle}{\langle U\rangle}=4 B^{2}=\left(\frac{\sqrt{2}}{\chi}\right)^{4 / 5}
$$


For negative $a$ (attractive interaction), a noncollapsing condensate can exist only for a maximum number of atoms. To find this critical number, we recall that there is no global minimum of the chemical potential at this critical value so the conditions are the vanishing of the first and second derivatives of the chemical potential

$$
\mu^{\prime}=\mu^{\prime \prime}=0
$$

This calculation gives $\chi_{c}=-0.76$. So we get for the critical number of atoms

$$
N_{0}^{c}=\frac{\chi_{c}}{a} \sqrt{\pi \hbar / m \omega}
$$

In terms of the oscillator length defined as $d=(\hbar / m \omega)^{1 / 2}, N_{0}^{c}=\sqrt{\pi} \chi_{c} \frac{d}{a}$

And if we substitute the values corresponding to lithium ${ }^{7} \mathrm{Li}$, namely, $a=-1.44 \mathrm{~nm}, d=3.13 \mu \mathrm{m}$, we get $N_{0}^{c}=2928$

\section{Thomas-Fermi limit}

In the Thomas Fermi limit the kinetic energy is ignored for large systems.

In this limit the chemical potential becomes

$$
\mu^{*}=\frac{3}{8 B}+\chi B^{3 / 2}
$$

Now we minimize $\mu^{*}$,

$$
\frac{d \mu^{*}}{d B}=0=-\frac{3}{8 B^{2}}+\frac{3}{2} \chi B^{1 / 2}
$$

And hence $B=(1 / 4 \chi)^{2 / 5}$.

In this case the chemical potential becomes

$$
\mu=\frac{5}{8}(4 \chi)^{2 / 5} \hbar \omega
$$

which is the first term of eq. (11)

\section{Conclusion}

In this work I studied the problem of Bose -Einstein condensation in atomic gases atultra low temperatures. The system is considered to be confined in an isotropic harmonic potential trap. A Gaussian wavefunction is used to solve the Gross-Pitaevskii equation to calculate the chemical potential of the gas. The solutions were discussed in the strongly as well as the weakly interacting limits.

Without an external trap the system of interacting bose atoms has a soliton solution (Drake Perez et al., 2005).

In a future work I should take this into account in the variational wavefunction.

\section{Acknowledgement}

I would like to thank Al al-Bayt University for a sabbatical year that was given to me which I spent at the Department of Physics of the University of Jordan.

\section{References}

Anderson, M. H., Ensher, J. R., Matthews, M. R., Wieman, C. E., \& Cornell, E. A. (1995). Observation of BoseEinstein condensation in a dilute atomic vapor. science, 269(5221), 198-201. http://dx.doi.org/10.1126/ science.269.5221.198

Baym, G., \& Pethick, C. J. (1996). Ground-state properties of magnetically trapped Bose-condensed rubidium gas. Physical review letters, 76(1), 6. http://dx.doi.org/10.1103/PhysRevLett.76.6

Bradley, C. C., Sackett, C. A., Tollett, J. J., \& Hulet, R. G. (1995). Evidence of Bose-Einstein condensation in an atomic gas with attractive interactions. Physical Review Letters, 75(9), 1687. http://dx.doi.org/10.1103/ PhysRevLett.78.985

Chase, K. C., Mekjian, A. Z., \& Zamick, L. (1999). Canonical and microcanonical ensemble approaches to BoseEinstein condensation: The thermodynamics of particles in harmonic traps. The European Physical Journal B-Condensed Matter and Complex Systems, 8(2), 281-285. http://dx.doi.org/10.1007/s 100510050691 
Davis, K. B., Mewes, M. O., Andrews, M. R., Van Druten, N. J., Durfee, D. S., Kurn, D. M., \& Ketterle, W. (1995). Bose-Einstein condensation in a gas of sodium atoms. Physical review letters, $75(22), 3969$. http://dx.doi.org/: 10.1103/PhysRevLett.75.3969

Drake Perez, J. C., Trallero-Giner, C., Lopez Richard, V., Trallero-Herrero, C., \& Birman, J. L. (2005). GrossPitaevskii equation: Variational approach. physica status solidi (c), 2(10), 3665-3668. http://dx.doi.org/10. $1002 /$ pssc. 200461762

DuBois, J. L., \& Glyde, H. R. (2001). Bose-Einstein condensation in trapped bosons: A variational Monte Carlo analysis. Physical Review A, 63(2), 023602. http://dx.doi.org/10.1103/PhysRevA.63.023602

Fetter, A. L. (2009). Rotating trapped bose-einstein condensates. Reviews of Modern Physics, 81(2), 647. http://dx.doi.org/10.1007/s 11490-008-1001-6

Margetis, D. (2015). Bose-Einstein condensation at finite temperatures: Mean field laws with periodic microstructure. arXiv preprint arXiv:1509.04364.

Mateo, A. M., \& Delgado, V. (2007). Ground-state properties of trapped Bose-Einstein condensates: Extension of the Thomas-Fermi approximation. Physical Review A, 75(6), 063610. http://dx.doi.org/10.1103/PhysRevA. 77.013617

Pethick, C. J., \& Smith, H. (2008). Bose-Einstein condensation in Dilute gases. Cambridge: Cambridge University Press. https//doi.org/10.1017/CBO9780511802850

Rogel-Salazar, J. (2013). The Gross-Pitaevskii equation and Bose-Einstein condensates. European Journal of Physics, 34(2), 247. http://dx.doi.org/10.1088/0143-0807/34/2/247

\section{Copyrights}

Copyright for this article is retained by the author(s), with first publication rights granted to the journal.

This is an open-access article distributed under the terms and conditions of the Creative Commons Attribution license (http://creativecommons.org/licenses/by/4.0/). 